\title{
DIE STUNDE DER WAHREN EMPFINDUNG. DIE ETABLIERUNG DER GRUNDKONSTITUENTEN DER NARRATIVEN VermitTlung beI PETER HANDKE
}

Anđelka Krstanović, Univerzitet u Banjoj Luci, kandelka@gmail.com

10.31902/fll.28.2019.1

UDK 821.112.2.09-32

Die Erzählung Die Stunde der wahren Empfindung wurde 1975 veröffentlicht. Sie weist biographische Merkmale auf - den Ehebruch, den Aufenthalt in Paris, die Erfahrung, Vater zu sein. Nach der Erzählung Wunschloses Unglück steht wieder das Ich des Autors im Fokus der existenziellen Überlegungen, die in Form eines literarischen Werkes aufgearbeitet werden.

In diesem Beitrag werden die narrativen Verfahren in der Erzählung nach Genettes Modell analysiert. Bei der Analyse des Zeitraumes werden zusätzlich die theoretischen Ansätze von Bachtin und Lotman einbezogen, bei der Verdeutlichung der Normen und Werte der Figuren Erläuterungen von Carola Surcamp zur Perspektivenstruktur. Der Einblick in die Narration soll die Etablierung der Erzählverfahren in den 70er Jahren verdeutlichen, die auch für spätere Werke von Peter Handke kennzeichnend sind.

Schlüsselwörter: Peter Handke, Authentizität, Fiktion, Narration, Perspektivenstruktur, Chronotopos, Intertextualität

1. Der Diskurs und die Handlung in Die Stunde der wahren Empfindung Die Erzählung beginnt mit einem symptomatischen Fragesatz, den ein extradiegetischer Erzähler einleitet. Die ganze Geschichte wird von dieser außenstehenden Stimme vermittelt. Dabei ist die Fokalisierung von der Stimme zu trennen, denn die Geschichte wird aus der Perspektive der Hauptfigur Gregor Keuschnig dargestellt, d.h. durch eine interne Fokalisierung. Der einleitende Satz offenbart zugleich die Stellung des Erzählers zu der Geschichte: „Wer hat schon einmal geträumt, ein Mörder geworden zu sein und sein gewohntes Leben nur der Form nach weiterzuführen?" (Handke 7)

Die Erzählinstanz präsentiert die Geschichte als ein außergewöhnliches Ereignis und beginnt sie deshalb in Form von medias res. Der Traum nämlich, der einen Schnittpunkt im Leben von Gregor Keuschnig darstellt, als Auftauchen eines lang andauernden Brütens der Unzufriedenheit in seinem Unbewussten, bezeichnet zugleich den Anfang eines inneren Prozesses, der allmählich die Umrisse einer bewussten Einsicht einnimmt. Gregor Keuschnig, Pressereferent der österreichischen Botschaft, der mit seiner Frau Stefanie und seiner vierjährigen 
Tochter Agnes in Paris lebt, findet nach diesem Traum sein bisheriges Leben unerträglich. Er wünscht sich plötzlich ein anderes Leben und erlebt den alltäglichen Ablauf, der seine Existenz bestimmt, als ein Vortäuschen des wahren Lebens. Die Kluft zwischen einem von den gesellschaftlichen Normen vorgeschriebenen Leben und einer authentischen Identität wird somit zum Thema dieser Erzählung, das vor der Motiv-Achse des Traumes entfaltet wird.

Das lineare Schema lässt sich durch drei innere Zustände verfolgen: der erste Traum, in dem Keuschnig als Mörder erscheint, den er als Bruch mit der Wirklichkeit erlebt, der Zwischenzustand, gekennzeichnet durch zwei Träume, der die Zerissenheit zwischen zwei möglichen Stellungen in der Welt offenbart, nämlich die Subjekt - oder Objektstellung, und zugleich zu der Einsicht führt, dass die Lösung in der Behauptung der eigenen Identität zu suchen ist, und schließlich der letzte Traum als eine Zusammenfassung der inneren Krisen mit dem Ausblick auf ein neues Leben mit einer freien Äußerung der eigenen Identität. Die Geschichte vermittelt ein extradiegetischer Erzähler, aber die Fokalisierung ist eine konsequent beibehaltene interne Fokalisierung der Hauptfigur, woraus sich die Subjektivität der Darstellung ergibt. Die Geschichte wird in einer chronologischen Anordnung vermittelt, mit Ausnahmen von einigen Erinnerungsrückblicken auf die Kindheit. Sie wird aber nicht durch die Darstellung der äußeren Geschehnisse entwickelt, die in einer kausal-logischen Verknüpfung zu verfolgen wären, sondern in die Gedanken der Hauptfigur verlegt. Es werden somit durchgehend die inneren Vorgänge im Bewusstsein der Hauptfigur dargestellt. Aus dieser Verlagerung der Geschehnisse auf das Innere ergibt sich eine weitere Konsequenz der Spaltung von der traditionellen Erzählweise, nämlich eine anders gestaltete Zeit, die in extremen Dehnungen zu erkennen ist.

Die Handlung verläuft als sukzessive Abfolge von inneren Wandlungen der Hauptfigur. Auf der Ebene der Frequenz reflektiert sich die Darstellung als ein Nacheinanderfolgen der Geschehnisse nach dem Schema „einmal geschehen, einmal erzählt“. Es wird auf diese Weise eine lineare prozesshafte Steigerung der inneren Wandlungen erreicht. Die gleichen Geschehnisse werden nicht mehrmals erzählt und sie werden auch nicht aus der Perspektive der anderen Figuren dargestellt. Die Konsequenz dieser Gestaltung ist ein subjektiver Blickwinkel, der den Leser intensiv in Keuschnigs Bewusstsein einweiht. Die Intensität der Vermittlung einer subjektiven Weltsicht wird zusätzlich durch die Erzähldauer erreicht, denn die Erzählzeit und die erzählte Zeit stimmen nicht überein. Während sich die Handlung auf zwei Tage Ende Juli erstreckt, nimmt die Erzählzeit einen Umfang von etwa 160 Seiten ein. Diese zeitliche Kluft verschafft genügend Raum, um die inneren Wandlungen von Keuschnig ausführlich verfolgen zu können.

Auf der Ebene des Modus wird die Geschichte abwechselnd durch den Erzählerbericht, die transponierte und dramatische Rede präsentiert. Die trans- 
ponierte und dramatische Rede tragen dazu bei, die Berichtpassagen, die durchgehend aus der Perspektive von Keuschnig vermittelt werden, glaubwürdig zu machen. Eine solche Konstellation des Modus verweist darauf, dass der ganze Fokus auf inneren Bewusstseinsprozessen von Keuschnig liegt.

\section{Aufbau der Figuren und Perspektivenstruktur ${ }^{1}$}

Neben der Hauptfigur Gregor Keuschnig treten in der Handlung auch einige Nebenfiguren auf. Sie werden entweder als namenlose Vertreter einer Klasse dargestellt (der Präsident, die Unbekannte, die Angestellte, der Schriftsteller) oder sie tragen die Eigennamen, so Keuschnigs Ehefrau Stefanie, die Tochter Agnes, die Geliebte Beatrice, die Freundin des Schriftstellers Françoise.

Die Hauptfigur wird am Anfang durch den Namen und einen detaillierten Bericht über ihren sozialen Status, Familienstand, die Wohnverhältnisse, Berufsangaben vorgestellt:

Damals, zu der Zeit, die noch andauert, lebte Gregor Keuschnig seit einigen Monaten als Pressereferent der österreichischen Botschaft in Paris. Er bewohnte mit seiner Frau und der vierjährigen Tochter Agnes ein dunkles Appartement im sechzehnten Arrondissement. Das Haus, ein französisches Bürgerhaus aus der Jahrhundertwende, mit einem steinernen Balkon an der zweiten und einem gußeisernen an der fünften Etage, stand neben ähnlichen Gebäuden an einem ruhigen Boulevard [....] (Handke 7)

An der Einleitung fällt auf, dass die äußeren formalen Merkmale die anfängliche Charakterisierung der Hauptfigur ausmachen. Dabei wird das Bürgerliche und Gesellschaftliche in den Vordergrund gestellt wie "Pressereferent" oder „Bürgerhaus", was mit einem konventionellen bürgerlichen Leben assoziiert wird. Keuschnig lebt unter der Obhut des bürgerlichen Lebens, hat einen ordentlichen Beruf, ist verheiratet, wohnt in einem bürgerlichen Haus.

Nach der Erläuterung des sozialen Status und Familienstandes wird an den Traum von Keuschnig angeknüpft, womit man die thematische Kluft zwischen Traum und Wirklichkeit in Gang setzt:

In einer solchen Nacht Ende Juli hatte Gregor Keuschnig einen langen Traum, der damit anfing, daß er jemanden getötet hatte [...] Welche Schande werde ich meinen Eltern bereiten, dachte er, während die Ermordete, eine alte Frau, dürftig vergraben in einer Holzkiste lag: ein

\footnotetext{
${ }^{1}$ Durch die Perspektivenstruktur werden Normen und Werte von Figuren und Erzählern vermittelt, wodurch die einzelnen Weltsichten zum Ausdruck kommen. Die Perspektivenstruktur ist daher von der Erzählperspektive streng zu trennen. Die Perspektivenstruktur ist nach Carola Surkamp „die Gesamtheit aller Beziehungen, die in multiperspektivisch erzählten Texten ... zwischen den Figurenperspektiven und der Erzählerperspektive bestehen." In: Ansgar und Vera Nünning (Hg.): Neue Ansätze in der Erzähltheorie, Bd.4, WVT, 2002, S. 234.
} 
Mörder in der Familie! Am meisten aber bedrückte ihn, daß er jemand andrer geworden war und doch weiter so tun mußte, als ob er dazugehöre. (Handke 8)

Der Bericht an dieser Stelle geht in das Gedankenzitat über. Der Traum wird von Außen als Anlass für eine Zustandsveränderung festgehalten und gleichzeitig werden diese Veränderungen aus der Innenperspektive von Keuschnig veranschaulicht. Er fühlt Scham, was auf Gewissensbisse hindeutet, und es wird ihm gleichzeitig die bittere Wahrheit bewusst, dass das weitere Leben zu einer Verstellung werden soll.

Die Charakterisierung der Figur wird an dieser Stelle auf die Wünsche verlegt, die aus dem Unbewussten schrittweise in das Bewusstsein eindringen. Keuschnig beginnt zu überlegen, wie er sich von seiner Frau trennen könnte:

Er wollte, sie würde ihn beim Aufwachen sofort fragen, was er denke, und er würde antworten:'Ich überlege gerade, wie ich dich aus meinem Leben wegdenken könnte.' Auf einmal wünschte er, nie mehr etwas von ihr zu hören oder zu sehen. Sie abtransportieren lassen. (Handke 11)

Die indirekte Rede wechselt mit dem Gedankenzitat, das die Gedanken unmittelbar vermittelt, und der Bericht fungiert als Erörterung der Richtung dieser Gedanken. Die darauf anknüpfende Passage mit der Beschreibung der Ehefrau zeigt noch einmal, dass der Erzähler die Perspektive von Keuschnig vermittelt:

Sie hatte die Augen geschlossen, schrumplige Lider, die sich jetzt manchmal spannten. Daran sah er, daß sie allmählich erwachte. Ab und zu gurgelte es durch ihren Bauch... Er schaltete den Plattenspieler aus, und sie öffnete die Augen. Mit offenen Augen sah sie jünger aus. Sie hieß Stefanie und gestern noch hatte sie ihn zumindest manchmal gerührt. (Handke 11)

Diese deskriptive Passage zeugt von der Entfremdung zwischen den Ehepartnern. Keuschnig nimmt an Stefanie nur physische Merkmale wahr, Augen, Lieder, Bauch, als ob sie für ihn ein fremder Gegenstand geworden wäre. Von der Entfremdung Keuschnigs zeugt auch die distanzierte Vermittlung, da die Frau mit „sie“, also in der dritten Person dargestellt wird.

Gregor erlebt einen tiefen psychischen Einschnitt und verharrt im Zustand der seelischen Leere. Es wird ihm klar, dass er sein bisheriges Leben nicht mehr leben kann, und ein anderes Leben zu leben, ist für ihn immer noch unvorstellbar:

Er zog die Stirnhaut herunter und schloß fest die Augen, als ob er das gefühllos gewordene Gehirn so wieder wärmen konnte. Ab heute führe ich also ein Doppelleben, dachte er. Nein, gar kein Leben: weder das ge- 
wohnte, noch ein neues; denn das gewohnte werde ich nur vortäuschen, und das neue wird sich erschöpfen müssen im Vortäuschen des gewohnten. Ich fühle mich hier nicht mehr am Platz, kann mir aber überhaupt nicht vorstellen, irgendwo anders am Platz zu sein; [...] Im nächsten Moment war ihm, als platzte er aus seiner Haut heraus, [...] (Handke 13)

Das ist die Stelle, die eine weitere Stufe der inneren Wandlung von Keuschnig festhält. Nach der Einsicht nämlich, in einem klischeehaften Leben nicht mehr verharren zu können, die in das Bewusste in Form des Traumes eindringt, kommt ein Zwischenzustand, der in Richtung einer klaren Vorstellung von einer anderen Lebensform zu entwickeln bleibt. Die Schwankungen und unklaren Vorstellungen werden durch häufige „als ob“ Sätze vermittelt, die auf Vorahnungen, aber keine klaren Gedankenumrisse hindeuten. Die extradiegetische Stimme hält den Leser in Spannung, da sie auf jegliche Kommentare und Urteile verzichtet. Es geht durchgehend um die ausführliche Darstellung des Bewusstseins von Keuschnig. Zu dieser Spannung trägt auch der Umstand bei, dass die Perspektive der anderen Figuren nicht vermittelt wird.

Keuschnigs Abneigung gegen ein klischeehaftes Leben, das sich vor allem in einer Kluft zwischen Sprache und Wirklichkeit offenbart, wird in den Passagen verdeutlicht, welche die Arbeit in der Botschaft darstellen, und ferner das Verhältnis zu Beatrice, seiner Geliebten.

Im Büro las er die Zeitungen, die jetzt erst angekommen waren. Es fiel ihm auf, wie oft in den Überschriften auf einer einzigen Seite stand:'Immer mehr [...]':'Immer mehr Babys werden überfüttert.' - 'Immer mehr Kinderselbstmorde.' Beim Lesen von TIME bemerkte er auf vielen Seiten den Satz 'I dig my life'. 'I dig my life', sagte ein Basketballstar. 'We are a happy family', sagte ein Kriegsveteran. 'I am very glad', sagte eine Countrysängerin. 'Now I dig my life', sagte ein Mann, der ein neues Haftpulver für sein Gebiß verwendete. (Handke 23)

Die klischeehaften Äußerungen, die ein wahres Leben vortäuschen und vorschreiben, werden durch die Medien als Maßstab auferlegt. In diesem Zusammenhang bezeichnet Manfred Mixner in seiner Monographie die Keuschnig fremd gewordene Welt als ein „begriffliches Bezugssystem" (223). Die von der Konsumgesellschaft auferlegten Rezepte für ein echtes Erlebnis werden als vorprogrammierte Lenkung des menschlichen Lebens verstanden.

Rolf Günter Renner stellt ferner in seiner Untersuchung zu Peter Handke im Zusammenhang mit der Thematisierung der Kluft zwischen einer wahren Identität und vorgeschriebenen Form des Lebens fest, dass hier die Wirklichkeit durch Sprache zum Klischee wird, und Keuschnig ein authentisches Erlebnis anstrebt, keine sozial kodierte Sprachformeln. (95) 
Eine weitere Passage, in der Keuschnigs Verhältnis zu seiner Geliebten veranschaulicht wird, hat die gleiche Funktion, nämlich das Gerüst der Einsicht im Traum durch zusätzliche Informationen zu fühlen. Nicht nur die Ehe als konventionelle Form wird zum Verhängnis, sondern auch alle anderen vorgespielten Bindungen.

Er dachte an den Kinderwagen ... und drehte sich unwillig weg, als ihm Beatrice wie üblich aus dem Rock helfen wollte. Er war es jetzt, der auf einmal Angst hatte, etwas Falsches zu sagen, etwas Falsches zu tun; er war es, der sich bei allem auf einmal etwas denken mußte, beim Fleischzerschneiden, bei einer Umarmung, sogar beim Atemholen. Was sich so vertraut ereignen sollte, vollführte er als zeremonielle Vorgänge, ängstlich bedacht, nicht aus der Rolle zu fallen. (Handke 28)

Diese Erfahrungen zeugen von der Form der Entfremdung von Keuschnig. Alle seine Handlungen betrachtet er als vorgespielt. Die zersplitterten Gegenstände um ihn haben an Sinn verloren. Er betrachtet sein bisheriges Leben als ein Rollenleben, in dem er Beamter, Ehemann, Geliebter ist, aber kein Mensch. Er schlüpft im Laufe seiner Verwandlung aus der Rolle der gestischen Figur ${ }^{2}$, in der die menschlichen Handlungen sozial verankert sind, und bekommt allmählich die individuellen Züge. Diese werden bildhaft in einem weiteren Traum offenbart, in dem er als Zeichen einer sich offen zeigenden Identität nackt erscheint. In diesem Traum hat Keuschnig den Wunsch, vor der ganzen Gesellschaft nackt zu erscheinen, worin sich die tiefe Wahrheit seiner Lebenslage offenbart. Die Einsicht wird erzähltechnisch durch den inneren Monolog vermittelt:

Der Traum ist wahr gewesen [...] Der Traum ist vielleicht mein erstes Lebenszeichen seit langem gewesen. Er hat mich warnen sollen. Er wollte mich umdrehen, wie jemanden, der lange auf der falschen Seite gestanden hat. Ich möchte die schlafwandlerischen Sicherheiten für den Wachzustand vergessen. Die Träume zu vergessen war immer leicht. Die Sicherheiten zu verlieren, wird anstrengend sein, weil mir täglich die selben Sicherheiten begegnen werden - die nur andre mir vorgeträumt haben. (Handke 35)

Der Traum offenbart sich somit als ein Lebenszeichen, als Warnung, als Zeichen, das Keuschnig aus seinem Schlafwandeln herausrütteln sollte. Der Traum erweist sich als wahrhaftiges Leben, Leben als Traum. Die umgekehrte Perspektive, die aus der neu gewonnenen Einsicht erfolgt, enthüllt zugleich eine

\footnotetext{
${ }^{2}$ Der Begriff „gestische Figur“ stammt von Bertolt Brecht. Das Sprechen, die Haltungen, Gestik und Mimik der gestischen Figuren sind nach Brecht gesellschaftlich verankert, sie enthüllen die gesellschaftlichen Beziehungen zueinander. Vgl: Bertolt Brecht: Schriften zum Theater, Hg. W. Hecht, Band VI, Berlin und Weimar, 1964, S. 225.
} 
weitere Stufe der Charakterisierung der Figur, die nun ihre Vorahnungen und Wünsche in feste Vorstellungen und Werte umfunktioniert.

Die Erzählstrategie auf der Ebene des Modus besteht durchgehend im Wechsel des Berichts mit der Gedankenwiedergabe im transponierten und dramatischen Modus. Dieses Wechselspiel mündet immer wieder im Bericht, der als Zusammenfassung der inneren Wandlungen der Hauptfigur fungiert. Hierzu ein Beispiel:

Er dachte immer nur an eines, wie ein Wüstling, wenn auch das Eine nicht das Loch einer Frau war, sondern das Unvorstellbare. Sah denn niemand sein obszönes Gesicht? Er verstand nicht, dass keiner ihm nach dem ersten Blick einen zweiten, speziellen zuwarf, daß keine Frau zu Seite schaute, wenn sie ihn erblickte. Doch, eine Frau hatte weggeschaut und angewidert das Gesicht verdreht. Vielleicht mußte er im Park neben einem Gebüsch stehen, damit mehr ihn so sahen? [...] Und abstoßend war nicht, daß er sich so zeigte, sondern daß die Leute sich nicht auch so zeigten. Er überlegte, wie alt er war,[...] So viel Zeit hatte er also schon verbracht! [...] Seit der letzten Nacht aber war etwas stehengeblieben. Es war unkenntlich, und er konnte sich nur davon abwenden. Eingeweiht sein war lächerlich geworden [...] Er sah verkochten Reis aneinanderklumpen, in einem Topf so groß wie die Welt. Der Schwindel war aufgeflogen, und er war entzaubert. (Handke 36-39)

Die kursiv angegebenen Sätze sind in der erlebten Rede wiedergegeben. Die erlebte Rede, welche die psychischen Krisen von Keuschnig beleuchtet, mündet in einer Zusammenfassung in Form des Berichts, der verdeutlicht, wie der Bruch mit der Wirklichkeit und die Entzauberung schrittweise in das Bewusstsein eindringen. Die zitierte Passage zeigt ferner, wie die außenstehende Stimme auf jegliche Kommentare und Urteile verzichtet, so dass die Wertung der Überlegungen und emotionellen Schwankungen der subjektiven Perspektive von Keuschnig gänzlich ausgeliefet ist.

Die Haltung des Erzählers zu der Geschichte und zur Charakterisierung der Figur ist durch den Modus zu verfolgen. Ein bloßes Registrieren von Gedanken, Gefühlen und Handlungen mündet in eine distanzierte Haltung. Mixner weist diesbezüglich auf die Stelle hin, wo Stefanie Keuschnig verlässt, und stellt fest, dass hier zum ersten Mal der Erzähler von Keuschnig abrückt. (225) Auf die Funktion des Modus verweist auch Renner, indem er auf die Beschreibungspassage als Distanzierungsform am Ende der Geschichte hinweist. (102)

Keuschnig irrt durch die Stadt und empfindet Ärger über das sinnlose Leben der anderen. Dass er an dieser vorprogrammierten Welt keine Beteiligung mehr empfindet, bringt er auch durch die Feststellung zum Ausdruck, alles aufzählen, sich aber an nichts erinnern zu können. (Handke 60) Die mechanische 
Wahrnehmung tauscht er für ein wahres Erlebnis um. Eine dieser Proben, ein anderes Leben zu führen, stellt sein Seitensprung mit der unbekannten Angestellten in der Botschaft dar. Sie hat keinen Namen, ist kein Träger eines sozialen Status, ist ebenso zu keinen Normen und Werten verpflichtet. Der Beischlaf mit ihr vollzieht sich durch keine aufgespielte interessengeladene Motivation, sondern aus einem puren Naturtrieb. Davon wird er auch seiner Frau erzählen, um sie absichtlich zu verletzen, da er sie für einen authentischen Austausch als untauglich erlebt. Die Loslösung von den auferlegten Beziehungen, die seine wahre Identität durch Ersatzidentitäten stören, verläuft auch durch den Bruch mit der Kindheit. Diese Befreiung vollzieht sich zu einem Teil im Traum, in dem emotionale Bindungen mit inzestuösen Anspielungen aufgearbeitet werden, wie im folgenden Abschnitt: „Der nächste Traum handelte von seiner Mutter, die in seinen Träumen immer lebendiger wurde. Er tanzte mit ihr, ziemlich eng, scheute sich aber, sie Körper an Körper zu berühren, so daß er fast neben ihr stand, während sie tanzten." (Handke 111)

$\mathrm{Zu}$ anderem Teil wird die Loslösung durch eine bewusste Hinterfragung der Folgen der auferlegten Werte und Deutungen in der Kindheit vollzogen:

Sie (die Welt) war nur entdeckt, was die Geheimnistuereien betraf, mit denen die einen ihre Gewissheiten gegen andre verteidigten, und es gab jedenfalls keine künstlichen Geheimnisse mehr, mit denen er erpresst werden konnte, weder ein Geheimnis der Heiligen Kommunion noch des Universums [...] (Handke 81, 82)

Keuschnig erlebt die gegenwärtige Umgebung als sinnlos. Die Haltungen der Menschen, die er beobachtet, versteht er als ein leeres Rollenspiel. Und dies treibt ihn zu einer weiteren Überzeugung im Prozess der inneren Reifung hin, nämlich zu der Feststellung: „Ich glaube nicht an Gott." (Handke 80) Diese Behauptung wird im dramatischen Modus wiedergegeben, was die Unmittelbarkeit und Glaubwürdigkeit hervorruft. Keuschnig nimmt die einzelnen Gegenstände als zusammenhanglose Dinge wahr, die Menschen in ihren vorgetäuschten Handlungen. Er kann sich in einer solchen verlogenen und sinnlosen Welt eine übergeordnete Ordnung, die religiös zu rechtfertigen wäre, nicht vorstellen. Zugleich wird sich Keuschnig durch eine solche Feststellung dessen bewusst, dass der Mensch als Subjekt in der Welt auf sich gestellt ist. Um diese Einsicht in einen produktiven Zustand umzubilden, braucht er der Rechtfertigung einer solchen Überzeugung, bzw. das Signal eines möglichen Weges für eine andere Lebensform, und die wird er auf einem Kinderspielplatz erleben:

Dann hatte er ein Erlebnis - und noch während er es aufnahm, wünschte er, daß er es nie vergessen würde. Im Sand zu seinen Füßen erblickte er drei Dinge: ein Kastanienblatt; ein Stück von einem Taschenspiegel; eine Kinderzopfspange. Sie hatten schon die ganze Zeit so dagelegen, doch auf 
einmal rückten diese Gegenstände zusammen zu Wunderdingen. - 'Wer sagt denn, daß die Welt schon entdeckt ist?' - [...] Ich habe an ihnen kein persönliches Geheimnis für mich entdeckt, dachte er, sondern die IDEE eines Geheimnisses, die für alle da ist! 'Was Namen als BEGRIFFE nicht vermögen, leisten sie als IDEEN.' Wo hatte er das gelesen? (Handke 82)

Auch an dieser Stelle werden die entscheidenden Einsichten im dramatischen Modus mit dem Bericht verknüpft, sie erscheinen hier in Form eines Gedankenzitats, kursiv angegeben. Keuschnig stellt bei der Beobachtung der drei Dinge fest, dass es nicht nötig ist, das Leben durch auferlegte Begriffe und vorgeschriebene Bedeutungen zu leben, vielmehr ist der wahre Sinn in den persönlichen Erlebnissen zu suchen. Sie machen das Leben aus und eröffnen als Idee die Möglichkeit einer persönlichen Gestaltung des Zusammenhangs. Die Passage endet in der erlebten Rede als Reflexion auf das unmittelbare Erlebnis. Die transponierte Rede bekommt somit die Funktion einer bewussten Auseinandersetzung mit der unmittelbaren epiphanischen Einsicht.

Keuschnig möchte sich unbedingt von Stefanie trennen, und das geschieht auch, denn Stefanie verlässt ihn an einem Morgen. Dadurch kommt es aber nicht zu einer endgültigen Lösung von der Familie, denn das Kind bleibt bei ihm. Er bleibt eine kurze Zeit zu Hause mit dem Kind:

Er ging in der Wohnung hin und her; hob Sachen auf, um sie wegzuräumen, und legte sie nach einiger Zeit an dieselbe Stelle zurück. Er ging, stockte, drehte sich um sich selber und dachte plötzlich, daß er in seiner Ratlosigkeit und seinem Mißmut eine Art von Tanz aufführte! - Er konnte jetzt an keinem Spiegel vorbeigehen, ohne sich darin zu betrachten. Angewidert drehte er sich von dem einen weg und betrachtete sich im nächsten. (Handke 119, 120)

Diese Passage ist im Bericht wiedergegeben. Sie stellt zugleich einen Stillstand in der Vermittlung der Gedankenwelt von Keuschnig dar, so dass der Erzähler seine Handlungen von außen verfolgt, die gleichzeitig die innere Lage reflektieren. Keuschnig hat Zwangsvorstellungen, spürt Angst und Unsicherheit. Stefanie ist nicht mehr da, und seine Handlungen zeugen von einer Desorientierung. Die extreme Selbstbezogenheit hindert inn daran, sich mit dem Kind zu beschäftigen. Er spielt eine Sicherheit vor, es ist aber eine Zwangssicherheit. Nachdem er mit dem Kind das Haus verlassen hat, irrt er durch die Stadt. Es kommt dabei aber zu einem authentischen Erlebnis. Im Gegensatz zu anderen Figuren, die ihm auf seinem Weg nicht zu einer wahren Identität verhelfen können, bietet ihm das Kind eine unmittelbare Einsicht an: „Freundschaftlich gebrauchte sie (Agnes) plötzlich Ausdrücke, die sonst nur er verwendete. Und in allem, in Wolken, in den Baumschatten, in Wasserlachen, sah sie GESTALTEN - die er schon längst nicht mehr wahrnahm [...]" (Handke 144) 
Das Kind bestätigt ihm seine Weltanschauung, durch einen unmittelbaren spontanen Kontakt mit der Umwelt den eigenen Vorstellungen und Ideen einen freien Lauf zu geben. Auf diese Weise kann die Außenwelt die Innenwelt beseelen, statt sie zwanghaft zu determinieren. Nachdem das Kind von Stefanie entführt worden ist, löst sich die letzte soziale Bindung, und er überlegt, sich das Leben zu nehmen. Schließlich entscheidet er sich für eine neue Lebensform, obwohl dieses Leben, wie Renner es in seiner Monographie zusammenfasst, die Fiktivität des neuen Bewusstseins darstellt, was der Erzähler durch die distanzierte Haltung am Ende der Geschichte veranschaulicht, und somit ein offenes Ende darbietet. (102) Dieser endgültige Entschluss, die Wiedergeburt von Keuschnig, ist das Ergebnis eines langwierigen Prozesses der Auseinandersetzung mit sich selbst, der erzähltechnisch durch die Beibehaltung der internen Fokalisierung unmittelbar und ausführlich vermittelt ist. Die anderen Figuren, die nur ab und zu in kurzen Sätzen in direkter Rede auftreten, erfüllen konsequent die gleiche Funktion, Keuschnigs Reflexionen über ihre Aussagen zu vermitteln. So die Aussage von Stefanie, die durchgehend die Äußerlichkeiten beschäftigen: „Warum fiel ihr nichts an ihm auf? 'Du bist schon angezogen', sagte sie (Stefanie) und nahm den Kopfhörer ab. In diesem Augenblick kam es ihm vor, er könnte sich hinknien und alles, alles sagen." (Handke 11)

Oder die Aussage von Beatrice, die eine Auseinandersetzung mit ihrer Existenz vortäuscht:

Sie dachten sich nie etwas dabei, daß sie zusammen waren. 'Wenn man sich nichts dabei denkt, ist das vielleicht ein gutes Zeichen', sagte Beatrice. Sie machte aus allem, was ihr begegnete, Zeichen; aber auch dort, wo andre Böses angedeutet sahen, bemerkte sie eine Bestätigung, daß bald alles noch besser würde. (Handke 27)

Ähnlich die Aussage des Präsidenten, von dessen gesellschaftlichen Rolle die unreflektierte Leistung gefordert wird: „Auf die Frage des Journalisten, ob ein bestimmtes Projekt nicht sinnlos sei, antwortete der Präsident:'Ich kann mir nicht erlauben, das, was ich tue, für sinnlos zu halten.' Keuschnig gefiel diese Antwort, und er schrieb sie auf." (Handke 71)

Das Innenleben der Nebenfiguren, ihre Gefühle und Schwankungen werden nicht dargestellt. Sie haben keine individuellen Merkmale, sondern werden als Träger eines Zwangsrahmens vermittelt, die alles Persönliche in Keuschnig zu ersticken drohen. Durch ihre formellen Existenzen verhelfen sie ihm gleichzeitig seine eigenen Wahrheiten zu definieren, und zwar dem Umfang entsprechend, den das oppositionelle Verhältnis in der Lebenseinstellung darbietet. Wenige Sätze, mit denen diese Figuren skizziert werden, offenbaren aus Keuschnigs Perspektive ihr existenzielles Verharren hinter einer klischeehaften, durch Gesellschaft und Sprache limitierten Existenz. Die Figur des Schriftstellers stellt hierzu eine Ausnahme dar. Nur er vermag hinter dieser dicken Kruste des Konventio- 
nellen die tieferen Einsichten zu vermitteln. Beim Besuch im Haus von Keuschnig sagt der Schriftsteller:

'Es graust mir, nach links und rechts zu schauen: überall giert schon etwas danach, gesehen zu werden. Schon wieder ein um den Hals gebundener Pullover, schon wieder der Holzkohlenrauch aus den Vorgärten. Bevor ich einmal jemanden traf, nahm ich mir vor, ganz auf ihn einzugehen - aber als ich dann vor ihm stand, dachte ich: Warum eigentlich? Und schaute lange überdrüssig in sein langweiliges Gesicht [...] Ich wundere mich, wie man in den Sternen Bilder sehen kann. Mir gelingt es nicht, jeweils einzelne unter den Sternen als SternBILDER zusammenzunehmen. So habe ich auch keine Idee, wie ich all die einzelnen Erscheinungen zu ErscheinungsBILDERN zusammenfassen sollte. Ist euch aufgefallen, wie oft manche Philosophen die Wörter 'versöhnen', 'bergen' und 'retten' verwenden? VERSÖHNT werden bei ihnen die BEGRIFFE; GERETTET werden die ERSCHEINUNGEN; und zwar von den BEGRIFFEN; und GEBORGEN sind die von den Begriffen geretteten Erscheinungen dann in den IDEEN. Ich kenne wohl die Ideen, aber ich fühle mich nicht in ihnen geborgen. Ich verachte nicht die Ideen, sondern diejenigen, die sich in ihnen geborgen fühlen - vor allem, weil sie dort vor mir in Sicherheit sind. Geht es dir ähnlich, Gregor? Wachst du nie auf, und es gibt den Zusammenhang nicht mehr?' (Handke 93)

Der Schriftsteller stellt hier das Sprachrohr des Inneren von Keuschnig dar. Er entzieht sich der banalen Alltäglichkeit, den fremden Begriffssystemen und zweifelt an dem vorgegebenen Zusammenhang. Diese Existenz steht im schroffen Gegensatz zu anderen Figuren, die gesellschaftlich determiniert sind und somit als Vertreter der gestischen Figuren auftreten. Der Schriftsteller fungiert als eine Außeninstanz, die, wo immer sie praktisch zu handeln beginnt, zum Scheitern verurteilt ist: „'Es ist seltsam', sagte der Schriftsteller und goß sich das Glas so voll, daß der Rotwein auf das Tischtuch lief [...] 'Der muß seinen Frieden mit der Welt gemacht haben', sagte der Schriftsteller, der sich gerade eine Zigarette in den Mund steckte und sie dabei abbrach." (Handke 93, 104)

Der Schriftsteller ist die Figur, die die Welt zu artikulieren vermag, worauf die verba dicendi hinweisen, mit denen die Figurenrede immer wieder ergänzt wird. Ganz anders zeigt sich das bei Keuschnig, dessen Reflexionen sich überwiegend im Bewusstsein abspielen, worauf die verba credendi hindeuten, bis auf wenige Stellen, wo er seine Einsichten zwar klar in Form eines Selbstgesprächs, aber ohne Bezug auf ein „Du“ artikuliert. Der Schriftsteller ist somit die einzige Figur, die mehr Sprachraum in Anspruch nimmt. Diese Figur bekommt die Züge einer sinnvollen Instanz, nämlich einer poetologischen, welche sich der Welt entzieht, um sie zugleich zu durchschauen. Am Ende der Geschichte hat 
der Schriftsteller über Keuschnig nichts mehr zu notieren, weil Keuschnigs Lebenswandel abgeschlossen ist, die Geschichte ist zu Ende. In einem hellblauen Anzug tritt Keuschnig in ein neues Leben ein, sich aller Risiken dieses Entschlusses bewusst. Er hat keine Ansprüche auf ein auferlegtes Zusammenleben, aber einen Anspruch auf die Selbstverwirklichung.

\section{Die intertextuellen Bezüge in Die Stunde der wahren Empfindung}

Die Erzählung enthält einige bedeutende intertextuelle Bezüge zu Prätexten. Sie weist aber keinen epigonenhaften Charakter auf, im Gegenteil, sie entfaltet vor der Folie der Vorlage eine anders beschaffene mögliche Welt. In diesem Zusammenhang könnte beispielsweise der Bezug zu Kafkas Erzählungen verfolgt werden. Manfred Mixner notiert dazu: "Wie Kafkas Gregor Samsa ist auch Handkes Gregor Keuschnig aus einer gewohnten/gewöhnlichen Lebenspraxis ausgeschieden, nur ist dieser Vorgang bei Handke nicht mehr sinnbildlich fassbar, ist keiner symbolischer Vorgang, sondern ein 'realistischer' Bruch [...]" (219)

Das Motiv des Traumes, bei Kafka öfters vorkommend, ist auch in Handkes Erzählung das Hauptmotiv. Der Name der Hauptfigur in Die Verwandlung ist identisch mit dem Namen der Hauptfigur in Die Stunde der wahren Empfindung. Auch die neue Situation teilen die Protagonisten, mit der sie nach dem Traum am Anfang der Erzählung konfrontiert werden. Bei Kafka wird der Traum von Gregor Samsa zur Wirklichkeit, eine traumhafte Lage entpuppt sich als Wirklichkeit. In der Stunde der wahren Empfindung ist der Traum nur ein Zeichen, das verhilft, die Wirklichkeit im Bewusstsein zu analysieren. Gregor Samsa verkommt in einer verständnislosen Umgebung, und diese psychische Verzerrung wird in einem grotesken Bild eines Ungeziefers nahegebracht. Die Materialisierung des Psychischen zeigt Gregors Ohnmacht und schließlich das Abfinden mit der Objektstellung in der Welt. Bei Gregor Keuschnig führt die Einsicht in die Entfremdung von der Umgebung zunächst zu einem Schwanken zwischen Objekt - und Subjektstellung. Für dieses Schwanken steht sein Traum, das er letztlich durch eine Auseinandersetzung mit sich selbst in die Behauptung seiner Subjektstellung umwandelt, ganz ungeachtet der ungewissen Zukunft. Die Ausprägung der eigenen Identität wird bei Handke als ein Prozess der inneren Reifung und einer bewussten Selbstbehauptung dargestellt, im Gegensatz zu Samsas Objektstellung, die durch Selbsttilgung unausweichlich zu einem Absterben führt, bzw. zur Aufgabe der Identität. Gregor Keuschnig verwirft die rollenhafte Zuneigung und eine Lieferung des Sinns durch die anderen und besteht auf der Veranlagung seines Selbst. So behauptet Gregor Keuschnig seine Subjektstellung am Ende der Geschichte, auch unter dem Risiko, dass diese mögliche Welt eine ungewisse Zukunft mit sich bringt. Denn alle anderen Lebensformen wären ein Leben der anderen, eine verlogene Geborgenheit. 
Der intertextuelle Bezug ist auch zu Schuld und Sühne von Dostojewski herzustellen. Das Motiv des Mordes an der alten Frau tritt in beiden Werken auf. Auch bei Raskolnikow startet der Konflikt mit der Wirklichkeit einen inneren Prozess der Wandlung wie bei Keuschnig. Bei Keuschnig fungiert aber die ermordete alte Frau als Traum, der vor der Wirklichkeit warnt, bei Raskolnikow wird sie zur Wirklichkeit. Dabei entfaltet sich für Raskolnikow der Bruch mit der Wirklichkeit zu einer ethischen Frage, bei Keuschnig zu einer existenziellen Frage. Für Keuschnig ist auch die Ethik ein auferlegter Rahmen, er glaubt nicht an Gott und erinnert sich daran, dass er in der Kindheit die Gebete formal ausübte, ohne dabei eine authentische Empfindung zu erleben. Bei Raskolnikow ist der Prozess ein umgekehrter, von einem Gottlosen wird er zu einem Gläubigen. Bei Keuschnig wird der Mord an der alten Frau im Bewusstsein ausgetragen und fungiert als Warnzeichen, das die soziale Umgebung als eine verbrauchte Form eines „es gehört sich so“ Lebens darstellt, wobei die alte Frau als Inbegriff aller vorgetäuschten Ordnungsformeln gilt. Keuschnig steigt aus diesem Rahmen heraus. Raskolnikows alte Frau fungiert als Inbegriff aller ungerechten Normen, die sozial determiniert sind, was sich zu einem bewussten Eingreifen in das ethisch Perverse entfaltet. Bei beiden Figuren setzt der innere Wandel in einer privaten Umgebung an, in der Wohnung, die als fremd und beengend empfunden wird und somit als gestimmter Raum die psychische Lage reflektiert. Während Keuschnig ferner die Lösung in der Trennung von allen Bindungen sucht, wird Raskolnikow durch Bindung erlöst, durch die Liebe zu Sonja, welche die Züge einer Auferstehung annimmt. Raskolnikows Natur wird als eine zerspaltene und unvollendete reflektiert, die zu ihrer Vollendung einen übergeordneten Rahmen benötigt. Ganz anders verhält es sich bei Keuschnig, der auch den religiösen Rahmen verwirft und das Schicksal eines auf sich gestellten Wanderers wählt. Er verzichtet auf eine Lenkung und stellt sich die Aufgabe, durch authentische Erlebnisse die ursprünglichen Wahrheiten wieder-zu-holen als Maßstab aller Dinge.

Bei dem thematischen Schwerpunkt der Kluft zwischen Ich und Welt erweisen sich auch Parallelen zu Thomas Mann. Sein Protagonist Thomas Buddenbrook musste auch die Bekenntnis ablegen, dass seine Existenz an eine Grenze gelangt ist, an der der Zwiespalt zwischen Ich und Wirklichkeit nicht mehr zu vertuschen war. Allerdings kommt er zu dieser Einsicht durch eine Außeninstanz, nämlich die Lektüre von Schopenhauer. Sein langer innerer Monolog im zehnten Teil der Buddenbrooks entlarvt den Umfang der langjährigen Vortäuschung eines Lebens. Keuschnig verhilft bei dieser Einsicht der Traum. Thomas Buddenbrook versteht die Individualität als ein Rollenspiel, durch Tradition und Religion auferlegt. Die Lösung sieht er, der Philosophie von Schopenhauer entsprechend, in dem Tod, wo es möglich wird, das Wesen von allen individuellen Fesseln zu befreien. Bei dieser tröstenden Einsicht, dass es doch die Erlö- 
sung gibt, wenn auch durch den Tod, sagt er: "Ich werde leben!" (Mann 659) Keuschnig zieht eine andere Lehre aus der Einsicht, dass ein Rollenleben nicht der ursprünglichen menschlichen Natur entspricht. Im Gegensatz zu Thomas Buddenbrook darf er es sich als Bürger des 20. Jahrhunderts leisten, auf jegliche vorprogrammierte Lebensformen, ob durch Religion, Sitten oder Gesellschaft determiniert, zu verzichten, und sogar einen metaphysischen Rahmen zu verwerfen. Er nimmt sich vor, die ursprünglichen Potenziale der Vielfalt seines Wesens in authentischen Erlebnissen hervorzurufen, und sagt bei dem entscheidenden Erlebnis auf dem Kinderspielplatz: „Ich habe eine Zukunft!“ (Handke 82)

Aus den genannten intertextuellen Parallelen lässt sich zusammenfassen, dass Handke die Prätexte wohl benutzt, und die intertextuellen Bezüge auch offen ins Erzählspiel bringt, allerdings nicht um eine epigonenhafte Schreibweise zu entwickeln, sondern um sie als Folie zu benutzen, vor der die eigenen Weltanschauungen als korrigierende Zukunftsweiser entfaltet werden.

\section{Der Chronotopos in Die Stunde der wahren Empfindung}

Der Raum in dieser Erzählung ist keine bloße topographische Größe im Sinne des Ortes der Handlung, sondern wird unmittelbar durch die Wahrnehmung von Keuschnig determiniert. Damit eng verknüpft wird auch die Zeit umfunktioniert und sie bildet mit dem Raum eine einheitliche semantische Größe. Am Anfang der Erzählung wird eine deskriptive Passage wiedergegeben, in der die Wohnung von Keuschnig beschrieben wird:

Er bewohnte mit seiner Frau und der vierjährigen Tochter Agnes ein dunkles Appartement im sechzehnten Arrondissement [...] Keuschnigs Wohnung war groß und verzweigt. Man konnte darin auf verschiedenen Wegen gehen und einander plötzlich begegnen. Der sehr lange Flur schien vor einer Wand aufzuhören - und ging dann, nach einem Knick, noch länger weiter, so dass man sich fragte, ob man immer noch in derselben Wohnung sei, [...] Die Wohnung war so verschachtelt, daß man das Kind, auch wenn es nicht verloren gehen konnte, häufig rief:'Wo bist du?' Das Zimmer des Kindes war von drei Seiten zu betreten [...] und ganz vorn, an der Straße, die 'Salons' [...] Die Wohnung kostete dreitausend Francs im Monat. (Handke 7, 9, 10)

Die Wohnung wird aus Keuschnigs Perspektive dargestellt, sie ist verzweigt und groß. Er empfindet sie in Form eines Labyrinths, in dem man nur schwer zueinander findet, und zugleich als einen dunklen Ort. Dieses Bild assoziiert ein Gefühl des Verlorenseins in der Wohnung. Ebenso fällt auf, dass die Wohnung sachlich beschrieben wird, mit formalen Einzelheiten, z.B. die Höhe der Miete. Mit keinem Wort wird die familiäre Idylle angedeutet, oder etwa Liebe, was die Entfremdung Keuschnigs von seiner Familie vorwegnimmt. Die 
gemeinsame Wohnung wird nur noch als ein befremdender Gegenstand erlebt. In einem schroffen Gegensatz zu dieser Wohnung steht der Außenraum, aus dem ab und zu durch den vorbeifahrenden Zug oder die Geräusche von Blättern der Platanen die Lebenszeichen hinein kommen. Dem Topos der statischen Wohnung ist die Dynamik der Straßen von Paris entgegengesetzt. Keuschnig läuft durch die Straßen von Paris und nutzt diesen Raum für seine inneren Auseinandersetzungen. Er hat aber auch eine besondere Auffassung von der Zeit auf diesen Straßen: „Die Zeit verging plötzlich zwischen den Häusern wie für ein außermenschliches System, in einem anderen Sinn, als die Straßen verliefen [...] Keuschnig erschien die Welt unter dieser unbarmherzigen Elementarzeit wie unbeseelt." (Handke 46)

Die Zeit hebt sich in seinem Erlebnis vom Raum ab und er empfindet hier die elementare Zeit als eine falsche und unbeseelte. Erst durch das Laufen in der Stadt ist er imstande, sich innerlich von dem langandauernden Rollenzustand fortzubewegen. Es scheint ihm, als ob die elementare quantitative Zeit, da sinnlos, und die qualitative verinnerlichte Zeit auseinanderklaffen würden:

[...] - und überall in der Stadt flimmerten unbenutzte Flipperautomaten, raselten und klingelten die benutzten, rauschten die Platanen und Kastanien an den Boulevards, schlingerten zwischen den Metrowaggons während der Fahrt die schwarzen Koppelschläuche, schauten einander Liebespaare in die Augen, lagen in den immer noch bestehenden WIMPYS die aufgeweichten Zwiebelringe unter den HAMBURGERS - und das alles [...] jahraus, jahrein mit derselben Unausweichlichkeit, Vorhersehbarkeit, Sterbenslangweiligkeit [...] (Handke 80)

An dieser Stelle wird die physische Zeit aus der Perspektive Keuschnigs nicht als ein Chronos der Wandlung und des Fortschritts erlebt, sondern als ein ewiges Kreisen des Sinnlosen. Das ewig wiederkehrende Gleiche, das sich simultan an verschiedenen Orten in Paris abspielt, lässt die Zeit zum Raum schrumpfen, zu einem zusammenhanglosen Stillstand.

Obwohl sich die inneren Krisen vor dem Hintergrund einer Großstadt entfalten, spielt die Natur immer wieder eine bedeutende Rolle. So empfindet Keuschnig den Regen als eine Erleichterung nach dem Druck des Konventionellen (Handke 73), oder er darf erleben, dass Stefanies Abschied an einem Morgen, an dem das Gewitter nach seinem Erwachen einsetzt, die Sonne mit sich bringt. Nachdem sie weggegangen ist, erscheint ein Regenbogen. (Handke 116) Die Naturerscheinungen, immer im Einklang mit seinem Inneren, sind durchgehend semantisch beladen.

Die Handlung erstreckt sich auf zwei Tage, und die Erzählzeit nimmt etwa 160 Seiten ein. Die Kluft zwischen Erzählzeit und erzählter Zeit hat zum Zweck, die inneren Krisen von Keuschnig intensiv zu vermitteln, so dass die Chronologie 
auf die Sukzessivität der inneren Wandlungen verlegt ist. Die Zeit nimmt somit die Züge einer inneren qualitativen Zeit an, die der chronologischen Außenzeit entgegengesetzt ist. Die innere Zeit wird auf die Abfolge der qualitativen Wand-

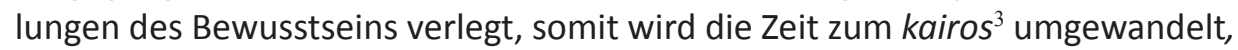
zu einer qualitativen Größe, im Gegensatz zur Außenzeit, die als chronos auf das bloße quantitative Zerfließen reduziert wird, und dadurch relativ und unbedeutend erscheint. Das qualitative Fortschreiten der inneren Zeit führt zu einer anders berschaffenen Einstellung zum Leben.

Die topographische Raumrelation Wohnung-Stadt fungieren als ein einander ergänzendes Paar. Während in der Wohnung die Prozesse im Bewusstsein durch Träume in Gang gesetzt werden, werden sie in der Stadt hinterfragt und aufgearbeitet. Es ist hierbei eine Lotmansche klassifikatorische Grenze ${ }^{4}$ zu erkennen. Sowohl die Wohnung als auch die Stadt sind semantisch als Formen eines vorgetäuschten Lebens vermittelt. Keuschnig steigt aber nicht aus diesem topographischen Raum heraus, z.B. durch einen Ortswechsel. Im Gegenteil, innerhalb von diesem Raum vollzieht sich der Entwicklungsprozess. Keuschnig überlegt an einer Stelle:

Im nächsten Moment, während er bewegungslos auf der Place Blanche stand, wollte er sofort von Paris wegfahren. Aber dann machte er sich klar, daß eine Reise vielleicht früher etwas geändert hätte - jetzt nicht mehr. Für das, was ihm zugestoßen war, gab es keine Fluchtmöglichkeit. Es war ihm auch nicht zugestoßen - es war eingetroffen. Er war schon lange fällig gewesen. San Diego und das Ballen der Fäuste: das bedeutete, daß er hier bleiben würde, und daß er noch nichts verloren gab. Ich werde es euch zeigen! dachte er. (Handke 42)

Diese Passage vermittelt, dass Keuschnig, obwohl in einem geschlossenen Rahmen verharrend, den festen Entschluss fasst, aus diesem nicht herauszusteigen, sondern sich innerhalb dessen mit seinem Selbst auseinanderzusetzen. Da er kein topographisches Entkommen wählt, verlegt er seinen Widerstand auf ein Laufen durch den inneren Raum, der die oppositionelle Linie zum Außenraum stellt. Die Dynamik der Wandlungen im Innenraum wird bildhaft durch das Laufen in der Stadt veranschaulicht. Somit ist die klassifikatorische Grenze im topologischen Paar Innen-Außen zu suchen und die Grenzüberschreitung voll-

\footnotetext{
${ }^{3}$ Vgl. Die Dissertation von Volker Schmidt Die Entwicklung der Sprachkritik im Werk von Peter Handke und Elfriede Jelinek aus dem Jahr 2007, in der der Ursprung, die Entwicklung und Ausprägung des Begriffs kairos in der modernen Literatur ausführlich behandelt wird.

${ }^{4}$ Unter Semantisierung des Raumes nach Lotman versteht man semantische Teilbereiche in einer Erzählung, die in einem oppositionellen Verhältnis stehen. Die klassifikatorische Grenze ist demnach die semantische Grenze, die seitens der Figur zu überschreiten wäre, um in einem Teilbereich die sujethafte Aufgabe zu vollenden, und damit die Spannung aufzuheben. Zu der Grenzüberschreitung siehe mehr in: Juri M.Lotman: Die Struktur literarischer Texte, 4. Auflage, W.Fink, 1993.
} 
zieht sich im Inneren von Keuschnig. Diese Grenzüberschreitung bedeutet, den Weg zur eigenen Identität zu finden.

Die Zeit und der Raum bilden dementsprechend eine Einheit, denn beide werden durch eine intensive existenzielle Auseinandersetzung mit dem eigenen Ich in den qualitativen inneren Zeitraum verlegt, und somit dem äußerlichen physischen Zeitraum entgegengesetzt.

\section{Schlussfolgerung}

Die Analyse des vorliegenden Korpus zeigt, dass Peter Handkes Erzählverfahren in den 70er Jahren festere Umrisse angenommen haben, die auch für spätere Werke konstituierend sind. Sie prägen sich in einem bestimmten Modus der Vermittlung der persönlichen Geschichten aus. Die Verweigerung der fiktiven Geschichten und das Herausfinden der passenden Erzälhmethoden für die Aufarbeitung von persönlichen Lebenserfahrungen ist ein Umstand, der für das ganze Opus des Autors kennzeichnend ist.

Die Stunde der wahren Empfindung ist eine Vorwegnahme der späteren narrativen Modelle in mehrerer Hinsicht. Sie sind erkenntlich in der Verlegung der Handlung in das Bewusstsein der Hauptfigur. Die lange Aufarbeitung des Status des Ich in der Welt macht die Handlung aus und überträgt sich auf extreme Dehnung der Erzählzeit. Die authentischen Erlebnisse des Ich, bei dem die Grenze zwischen Autor und Figur sehr fließend ist, werden durch ein monoperspektivisches Erzählen vermittelt, wobei ein allumfassender Blick auf die Welt verweigert wird. Das Bewusstsein der Hauptfigur steht im Fokus und die restlichen Figuren werden reduziert. Das literarische Werk dient zur Aufarbeitung einer privaten Erfahrung und wird als Ergänzungsfragment bei der Konstituierung der eigenen Identität erfasst, was narrativ mit offenem Schluss markiert wird. Die Entwicklung einer individuellen Existenz wird durch zwei Voraussetzungen determiniert. Zum einen ist das die Trennung von allen auferlegten sozialen Rollen, zum anderen ist diese Trennung mit der Sprachproblematik eng verknüpft. Denn die gesellschaftlich determinierten Rollenmechanismen werden durch Sprache auferlegt und sie werden entblößt. Nachdem die Figuren dieses sprachliches Befreiungsstadium erreicht haben, setzt der Individualisierungsprozess ein. Er spiegelt sich in der Herstellung eines unmittelbaren persönlichen Bezugs zur Welt, bei dem die individuellen Empfindungen von der Welt durch das Bewusstsein der Figur artikuliert werden. Das Herausfinden einer persönlichen Sprache und die treffende Artikulation der inneren Vorgänge sind dabei ein entscheidendes Anliegen. Die unantastbare Individualität wird nicht nur in diesem Werk beschworen, sondern auch in späteren Werken unterstrichen. Sie lässt sich niemals durch ein begriffliches System gänzlich definieren, man kann sich ihr durch deskriptive Passagen von inneren Prozessen im Bewusstsein, die auf Empfindungen basieren, nur annähern. Somit ist der Titel des behandelten Werkes eine Vorwegnahme dieser Ich-Position. 
Die wahre Empfindung als Maßstab einer authentischen Existenz ist ferner mit der Behandlung des Zeitraumes eng verknüpft. Wie diese Erzählung belegt, werden die Zeit und der Raum als physische Größen verworfen und als qualitative Variablen in das Innere der Figur platziert.

Nicht zuletzt ist für das Opus des Autors die Intertextualität kennzeichnend und sie ist auch in dieser Erzählung ein integrierter Teil der Geschichte. Die Prätexte werden aber nicht nachgeahmt, sondern im Prozess der Konstituierung einer authentischen Existenz hinterfragt und in Bezug auf die eigene Weltsicht korrigiert und erweitert.

\section{Literatur:}

Bachtin, Michail M. Chronotopos. Frankfurt am Main: Suhrkamp, 2008.

Brecht, Bertolt. Schriften zum Theater. Hg. W. Hecht. Band VI. Berlin und Weimar: Aufbau, 1964.

Dostojewski, Fedor Michailovitsch. Schuld und Sühne. Klagenfurt: Neuer Kaiser-Verlag, 1994.

Handke, Peter. Die Stunde der wahren Empfindung. 12. Auflage, Frankfurt am Main: Suhrkamp Verlag, 2012.

Kafka, Franz. Die Verwandlung. Frankfurt am Main: Suhrkamp, 1999.

Lotman, Juri M. Die Struktur literarischer Texte. 4. Auflage, München: W.Fink, 1993. Mann, Thomas. Die Buddenbrooks. Frankfurt am Main: S. Fischer, 1997.

Mixner, Manfred. Peter Handke. Kronberg: Athenäum Verlag, 1977.

Renner, Rolf Günter. Peter Handke. Stuttgart: J.B.Metzlersche Verlagsbuchhandlung, 1985.

Schmidt, Volker. Die Entwicklung der Sprachkritik im Werk von Peter Handke und Elfriede Jelinek. Univ. Diss. Heidelberg, 2007.

Surcamp, Carola. „Narratologie und possible-worlds theory: Narrative Texte als alternative Welten." Neue Ansätze in der Erzähltheorie. Hg. Ansgar und Vera Nünning. Bd.4, WVT: Trier, 2002. 153-185.

\section{ЧАС ИСТИНСКОГ ОСЈЕЋАЈА. ЕТАБЛИРАЊЕ ОСНОВНИХ КОНСТИТУЕНАТА НАРАТИВНОГ ПОСРЕДОВАЊА КОД ПЕТЕРА ХАНТКЕА}

Приповијетка Die Stunde der wahren Empfindung (Час истинског осјећаја) објављена је 1975. године. Прожета је биографским елементима - развод, боравак у Паризу, искуство очинства. Након приповијетке Wunschloses Unglück личностаутора поново долази у фокусегзистенцијалних промишљања, која се пласирају у форми књижевног дјела. 
У раду се анализирају наративни поступци у приповијеци према Женетовом моделу. Код анализе хронотопа додатно су укључене теоретске основе Бахтина и Лотмана а у дијелу у коме се представљају норме и вриједности фигура појаснили смо структуру перспективе. Увид у нарацију има за циљ да освијетли етаблирање наративних поступака у току 70их година, поступака, који су карактеристични и за каснија дјела Петера Ханткеа.

Кључне ријечи: Петер Хантке, аутентичност, фикција, нарација, структура перспективе, хронотопос, интертекстуалност. 